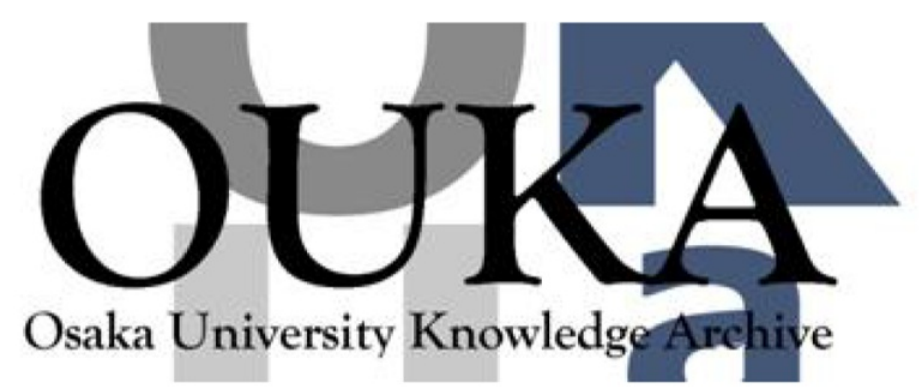

\begin{tabular}{|c|l|}
\hline Title & $\begin{array}{l}\text { Modular components for an optical array logic } \\
\text { system }\end{array}$ \\
\hline Author(s) & Tanida, Jun; Ichioka, Yoshiki \\
\hline Citation & Applied Optics. 26(18) p. 3954-p.3960 \\
\hline Issue Date & 1987-09-15 \\
\hline oaire:version VoR \\
\hline URL & https://hdl. handle. net/11094/2963 \\
\hline rights & \\
\hline Note & \\
\hline
\end{tabular}

Osaka University Knowledge Archive : OUKA

https://ir. Library. osaka-u. ac. jp/

Osaka University 


\title{
Modular components for an optical array logic system
}

\author{
Jun Tanida and Yoshiki Ichioka
}

\begin{abstract}
On the basis of modularization of an optical parallel array logic system (OPALS), a new concept of an optical digital computer is considered. The OPALS is a parallel digital optical computing system using optical array logic capable of executing parallel neighborhood logical operations, or cellular logic. Modularizing functional elements in the OPALS, stability, reliability, and flexibility of the system can be improved. Performance of the modularized OPALS is estimated.
\end{abstract}

\section{Introduction}

In today's information-oriented society, the great demand for information processing systems has led to the development of ultrahigh speed electronic computers and supercomputers. However, the switching speed of logic elements appears to be close to the physical limit, and data communication among logic elements becomes more and more difficult as switching speed and spatial density of logic elements increase. These problems might be solved by optical computing and communication techniques utilizing the great capability of light in information processing and in data communication. Thus light is regarded as a useful information carrier in the next generation of fast information processing systems. Many researchers are working to achieve optical computing systems. ${ }^{1-7}$

Several proposals for optical computers have already been presented ${ }^{1,2,5,6}$ using the parallel nature of light propagation. We have proposed the OPALS (optical parallel array logic system). ${ }^{7}$ Although these proposed optical computer concepts exhibit originality and have great potential, a number of problems must be solved to make such optical computers competitive with today's electronic computers. The problems are those of applicability to a wide range of computing problems, reliability, flexibility, controllability, and so on.

Fortunately, optics has a long history progress. Thus many techniques and devices can be used for

The authors are with Osaka University, Department of Applied Physics, 2-1, Yamadaoka, Suita, Osaka 565, Japan.

Received 17 February 1987.

0003-6935/87/183954-07\$02.00/0.

(C) 1987 Optical Society of America. constructing optical computers. Optical functional devices, such as spatial light modulators, ${ }^{8}$ can be used as 2-D switching arrays or image memories. Optoelectronic devices in microoptics, such as optical ICs (integrated circuits), laser diodes, and photodiodes, facilitate developing primitive elements for composing optical computers. In addition, 3-D LSIs (large scale integrated circuits), the next target of electronics development, ${ }^{9}$ would also be useful in optical computing systems.

In this paper, we present one direction for making an optical digital computer based on modularization of the OPALS. In Sec. II we show the system concept of the OPALS, and in Sec. III we consider problems to be solved in developing an optical computer. In Sec. IV we propose the concept of an optical digital computer based on the OPALS. In Sec. V we estimate the performance of the proposed optical computer.

\section{Optical Parallel Array Logic System}

The OPALS ${ }^{7}$ is a parallel optical digital computing system using optical array logic ${ }^{10}$ as its operational basis. Optical array logic can achieve parallel neighborhood logical operations, or cellular logic, ${ }^{11}$ for $2-D$ binary images, such as

$$
c_{i j}=R\left(\mathbf{a}_{i j}, \mathbf{b}_{i j}\right) \quad(i, j=1, \ldots, N),
$$

where $\mathbf{R}\left(\right.$ ) is the logical function, $\mathbf{a}_{i j}$ and $\mathbf{b}_{i j}$ are vectors consisting of values of the $i j$ pixel $\left(a_{i j}\right.$ and $\left.b_{i j}\right)$ and their neighbors $\left(a_{i-1 j-1}, a_{i-1 j}, \ldots\right.$ and $\left.b_{i-1 j-1, b_{i-1 j}, \ldots}\right)$ in input images $A$ and $B$, respectively, and $c_{i j}$ is the value of the $i j$ pixel in the output image. The OPALS executes any parallel neighborhood operation for 2-D binary images expressed by Eq. (1).

Figure 1 shows the processing procedures of optical array logic. Its processing principle is the same as that of array logic ${ }^{12}$ in electronics except for the parallelism. Namely, an operation for the specific $i, j$ in Eq. (1) is expressed by the logical sum of several product terms 


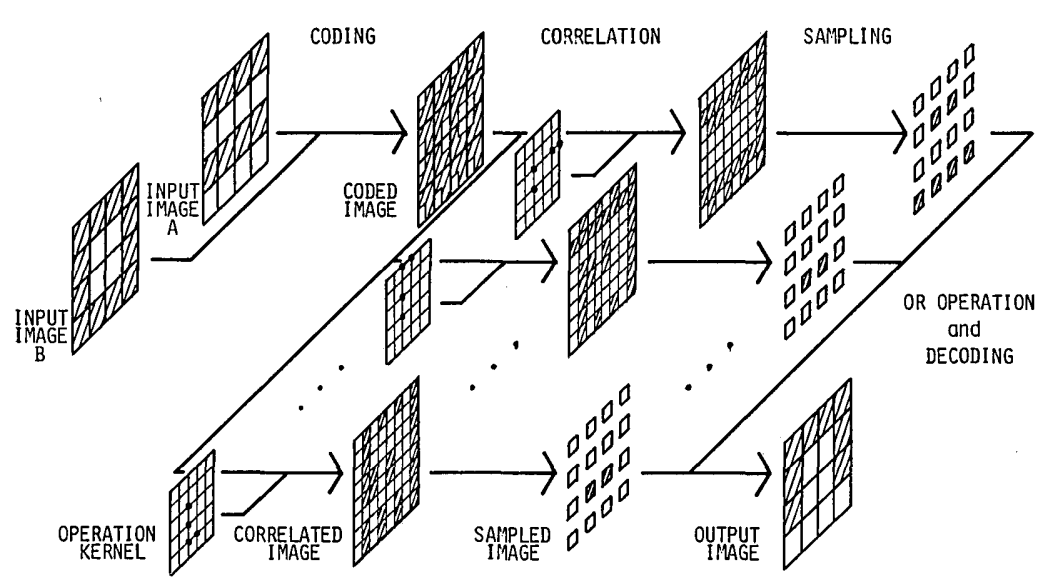

Fig. 1. Processing procedures of optical array logic.

and by a product term operation and oR operation. In this procedure, $N^{2}$ logical operations are concurrently executed for a given image.

As shown in Fig. 1, a product term operation is achieved by 2-D correlation of a coded image and an operation kernel followed by a decoding process. The decoding process consists of spatial sampling and thresholding. Although we have not clearly pointed out this thresholding process in previous papers, if the role of a photosensitive device is merely to detect whether light comes to it, this thresholding process will be implicit. The OR operation for the decoded signals provides the result of the given operation. This result is inverted and spatially expanded to match the data format in the next step. The type of operation is established by the combination of operation kernels selected. To simplify the description of the operations, we use symbolic notation. ${ }^{10}$ Programming of optical array logic will be described in a subsequent paper.

Optical array logic is a technique implementing parallel processing of two input and one output 2-D signals. Feeding the output signal back to the input port as one of the input signals in the next step of operation enables us to construct a simple iterative processing system. A system capable of implementing these operations is the OPALS. Figure 2 shows the schematic diagram of the OPALS. Its salient features are the capability of implementing fully 2-D parallel operation, programmability, the capability of iterative processing, and separability into modules. These features indicate the potential of the OPALS for constructing an optical digital computer.

\section{Problems of the OPALS as an' Optical Digital Computer Concept}

To use the OPALS as a basic element of an optical digital computer, many problems must be considered. In this section we discuss these problems.

\section{A. Optical Functional Devices}

Optical functional devices, such as spatial light modulators, ${ }^{8}$ are needed to make an OPALS. As shown in

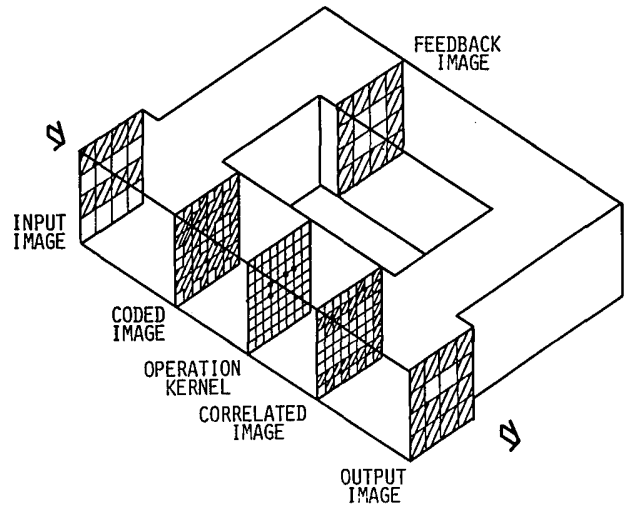

Fig. 2. Schematic diagram of the OPALS.

Fig. 1, optical array logic treats more than three 2-D variable data: input images $A$ and $B$, and operation kernels $1, \ldots, N$. Therefore, to implement optical array logic, some optical functional devices capable of writing/erasing $2-D$ patterns in real time must be used. The operating mode of these devices may be serial or parallel. However, to preserve the parallel nature of optical signals on the OPALS, optical functional devices operating in a parallel mode are much more desirable than those in a serial mode.

Although spatial light modulators at the present time do not have sufficient response speeds, spatial resolution, or energy consumption, their parallel operation in writing/erasing has served to demonstrate the capability of optical computing systems. However, much effort is needed to improve the characteristics of these spatial light modulators to construct optical computers competitive with electronic ones.

Another useful device is an optoelectronic hybrid device manufactured by 3-D LSI techniques in electronics. ${ }^{9}$ Although a hybrid device is inferior to an alloptical device in switching speed and energy efficiency, ${ }^{1}$ the processing flexibility and ease of fabrication in electronics are attractive. As described in Sec. IV.A, pixel-divided processing enables us to avoid the communication problem in electronics, namely, if one elec- 
tronic processing element processes only one datum concerned with a specific pixel, complicated connection lines are not needed. Communication among processing elements is achieved by optical interconnections. Processing elements on a substrate operating in parallel must be developed. However, future fabrication techniques in LSI devices facilitate production of such a device. This 3-D optoelectronic hybrid device can also be useful for optical computers.

\section{B. Stability and Reliability}

Stability and reliability are necessary for practical use of optical computers. At the present stage an optical computer system constructed with various optical components (lenses, mirrors, etc.) on an optical bench in a laboratory is suitable for both assembling a system and examining its operation. However, such a system requires delicate adjustment and isolation from external disturbances. As a result, the systems are unstable and unreliable.

One way to achieve stability and reliability is to package all optical hardware in a module such as an electronic IC but without loss of spatial dimension. Although technical difficulties exist, the concept of an optical functional module or 3-D optical IC is needed for optical computers sooner or later. Note that electronic computers are based on highly developed packaging techniques such as printed circuit boards and LSIs.

\section{System Controlling}

The OPALS executes processing in accordance with programmed operation kernels, so that the method of feeding the operation kernels into the optical system holds the key to efficient processing on the OPALS. As mentioned in Sec. III.A, operation kernels are 2-D pattern data, so writing/erasing in parallel is preferable. Thus operation kernels should be stored in a 2-D program memory and fetched in a sequence.

Here, another type of controlling method is considered, in which output processed by the OPALS is used as an operation kernel of the next processing step. This method involves a type of feedback and is expected to be a new type of controlling method.

\section{Efficient Applications}

The prospect of efficient applications motivates the development of optical computers. We consider two effective uses of optical computers.

One is to execute algorithms using global data communication such as the Fourier transform. Although this processing can be done by an electronic computer, an optical computer using optical interconnections will process such algorithms much more easily and simply than an electronic computer. Communication by photons in free space facilitates global interconnection and multiple fan-in and fan-out.

Another use of optical computers is to implement highly reliable processing by redundant processing paths, utilizing the parallel nature of optical computers. Most of the optical computers have SIMD (sin-

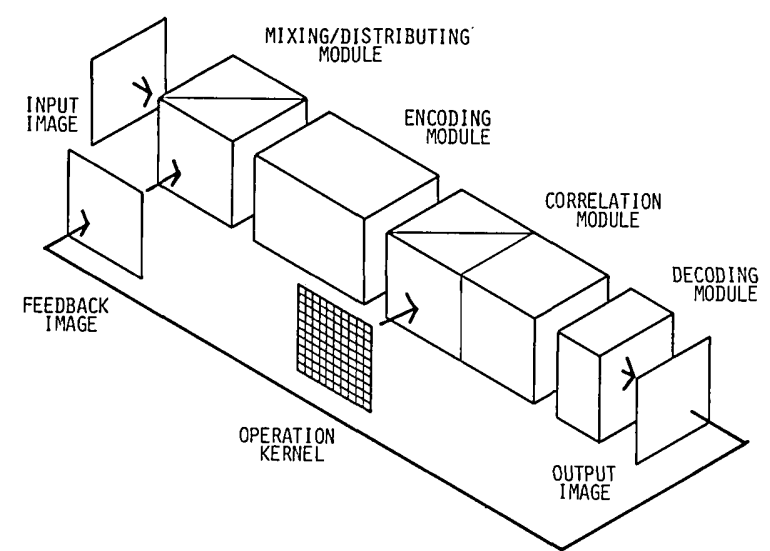

Fig. 3. Concept of the modularized OPALS.

gle-instruction stream multi-data flow) architecture because the parallel nature of optical systems is essentially space-invariant. Thus applications for SIMD machines must be considered, although limitations due to the SIMD architecture cannot be overcome.

Here, we propose an efficient use suitable for SIMD architecture. The proposed method is as follows: A datum to be processed is duplicated, loaded into all or a part of the processing elements in a 2-D register of an optical computer, and processed in parallel. Hence many copies of the output will be obtained. By applying majority logic to the outputs, high reliability is expected even if an operating error has occurred during processing in the several processing elements. Here, majority logic means a logical system assigning logical one to the output when the majority of the input is logical one. By sending different data into a 2-D register and processing in parallel, different kinds of results in various conditions can be obtained at one time.

\section{Modularized OPALS}

Now we describe a method of developing an optical computer based on the OPALS and show a concept of one version of a future digital optical computer. Although several technical difficulties still exist, the concept is worth pursuing because of its attractive features.

\section{A. General Concept}

The basic idea of constructing an optical digital computer is modularization of the OPALS, using separable functional elements in the OPALS. The OPALS can be constructed from several modules: mixing/ distributing, encoding, correlation, and decoding modules as shown in Fig. 3. For a set of these functional modules, the formats of input and output signals (for example, data arrangement, carrier wavelength, etc.) are matched to each other, so that the sets of functional modules can be connected freely.

Mixing/distributing, encoding, and decoding modules are composed of pixel processing elements as shown in Fig. 4. A pixel processing element processes only one pixel or a set of pixels in the same location $(i, j)$ 


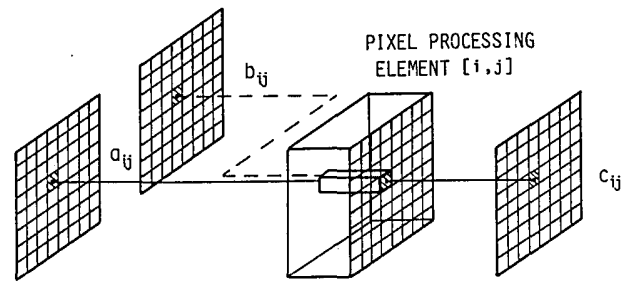

Fig. 4. Pixel-divided processing.

in the input images $A$ and $B$, e.g., $a_{i j}$ or $\left(a_{i j}\right.$ and $\left.b_{i j}\right)$; we call this pixel-divided processing. The advantages of pixel-divided processing are the easy change of pixel number and the capability of using LSI fabrication techniques. Appending pixel processing elements is possible by increasing the number of processing pixels, which can be done by duplicating a pixel processing element.

Processing on the system is controlled by operation kernels fed into the correlation module. The correlation module has two input ports, for a coded image and for an operation kernel, and performs correlation on them in real time. Using the correlation module a processed output image can be assigned as an operation kernel for controlling another OPALS. This controlling method is mentioned in Sec. III.C.

The most remarkable feature of the modularized OPALS is that various types of optical processing system can be constructed simply by changing the connections of functional modules. Therefore, the OPALS can be designed for a given problem.

\section{B. Mixing/Distributing Module}

The mixing/distributing module in Fig. 5 is used for shuffling two 2-D image data, and the resultant 2-D shuffled image is used for the input signal of the encoding module. This module is needed to implement pixel-divided processing. If a 2-D image signal is passed through this module in the reverse direction, it is divided into two 2-D images.

Figure 6 shows three methods of making a mixing/ distributing module. In Fig. 6(a) bundles of optical fibers, in Fig. 6(b) a polarized beam splitter and a grating, and in Fig. 6(c) a grating mirror are used, respectively. These mixing/distributing modules can be easily produced using conventional techniques for fabricating optical elements.

\section{Encoding Module}

The shuffled image obtained by a mixing/distributing module is converted into a coded image for optical array logic in Fig. 1 by an encoding module. Figure 7 illustrates an encoding module consisting of pixel encoding elements.

Since the data flow in the OPALS is synchronous, latch elements are desirable in the encoding module to maintain reliability of the operation. However, this does not mean special devices are needed for latching. If reliable operation is guaranteed, delay elements serve as latch elements. ${ }^{13}$ From the point of the view of cascadability, wavelength matching between input

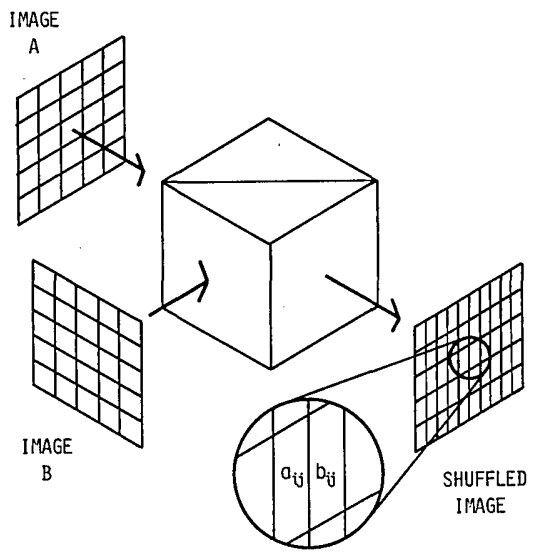

Fig. 5. Mixing/distributing module.

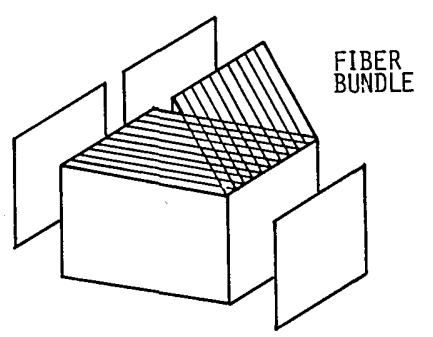

( a )
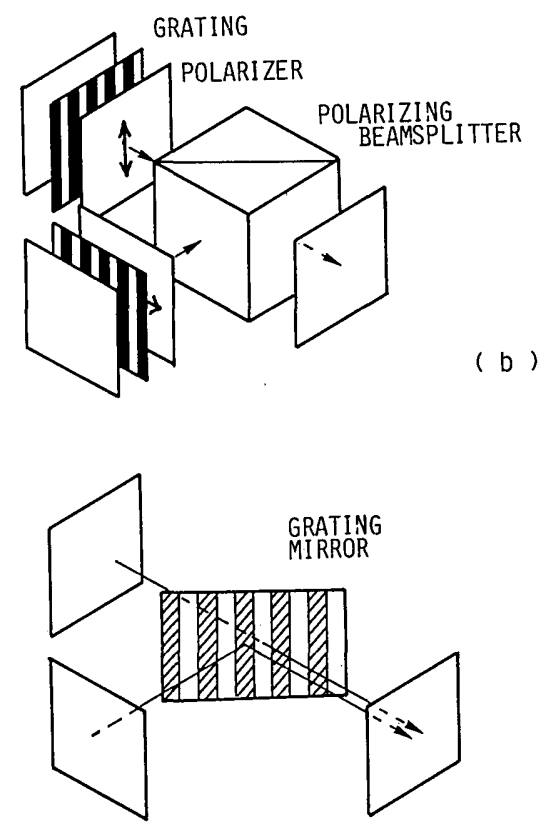

( c )

Fig. 6. Methods of the mixing/distributing module: (a) bundles of optical fibers, (b) polarized beam splitter and grating, (c) grating mirror.

and output signals and amplification in the encoding module are needed.

Various optical functional devices can be used for the encoding module. However, as discussed in Sec. III.B, these optical functional devices should be packaged for maintaining stability and reliability. Thus optoelectronic hybrid devices constructed by 3-D LSI technology will be better than all-optical devices such 


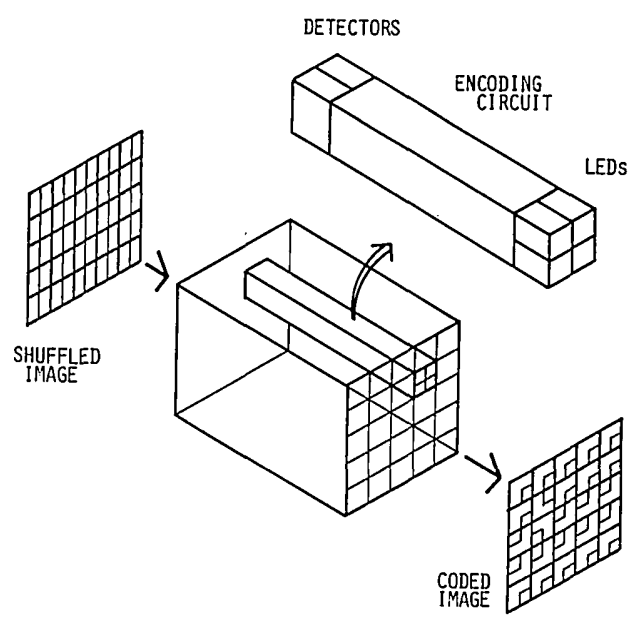

Fig. 7. Encoding module.

as spatial light modulators in the near future. Alloptical devices will follow hybrid ones.

\section{Correlation Module}

The correlation module shown in Fig. 8 performs the real-time 2-D correlation needed for optical array logic. This correlation is a specialized one because one of the operands, i.e., an operation kernel, is a pointwise function. To perform it, specially designed correlators are needed to prevent dispersion of optical power. Figure 9 is an example of such a correlator, i.e., a controllable multifocus imaging system, ${ }^{7}$ which is packaged in the correlation module.

This type of optical functional module is different from an optical IC constructed on a planar substrate. It uses lightwave propagation in free space for making good use of the parallel nature of optical processing without losing spatial dimension. Although this type of optical IC has still not been developed, it is necessary for realizing an optical computer.

The loading of the operands is a primary functional feature of the correlation module. As described in Sec. IV.A, both the coded image and operation kernel are put into the correlation module as 2-D images, so that identification of these two input images is needed. This can be achieved with photorefractive material, such as bismuth silicon oxide, ${ }^{8}$ for an optical shutter array. In this case, the switching configuration of the optical shutter array corresponds to the pattern of an operation kernel. The pattern is recorded on the photorefractive material at one wavelength. If a longer less-energetic wavelength of light is used for correlation, the desired correlation can be executed without disturbing the switching configuration of the optical shutter array. This switching configuration can be erased and rewritten, and hence variable correlation operations controlled by a 2-D optical signal can be implemented.

\section{E. Decoding module}

To complete the optical array logic process, an OR operation and decoding procedure are necessary.

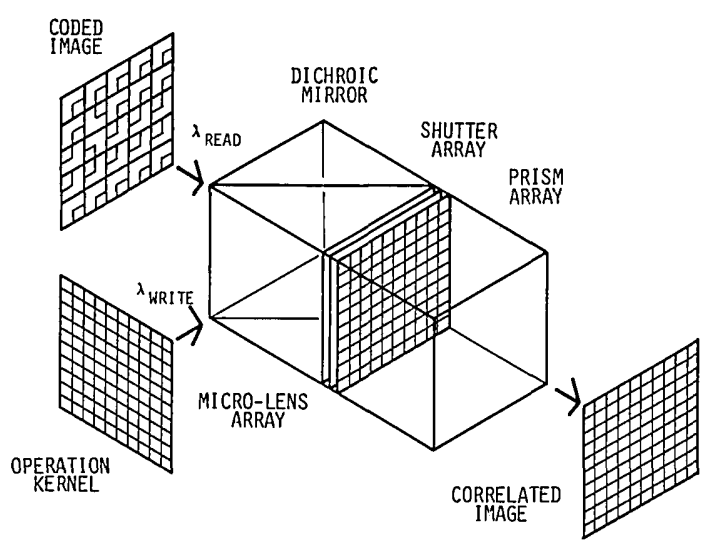

Fig. 8. Correlation module.

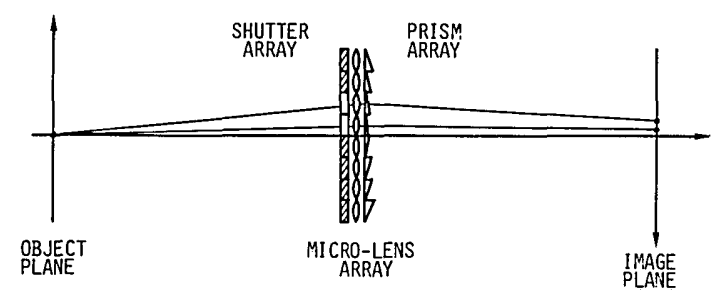

Fig. 9. Controllable multifocus imaging system.

These consist of spatial sampling, thresholding, oR operation, inverting, and spatial expansion of all pixel signals. Although these procedures appear to be troublesome, their implementation is not difficult. Spatial sampling is achieved by a simple sampling mask called the decoding mask. ${ }^{10}$ Thresholding can be neglected when using a photosensitive device which only detects the presence of light. The oR operation and inverting are combined into one operation called inverted-OR, or NAND. Spatial expansion, i.e., conversion of any quarter size of the sampled signal into a full size pixel, can be carried out with the pixel-divided diffusing box as shown in Fig. 10(a).

Two methods of implementing optical array logic are considered depending on whether multiple products are executed using sequential or parallel modes. In the sequential mode, product term operations needed for a given processing operation are performed time sequentially, and the oR operation is carried out at every time interval in which a product term operation is executed. In the parallel mode, multiple product term operations are executed at one time and the $O R$ operation is carried out after that. The decoding procedure depends on the method used. Figures 10(b) and (c) illustrate the difference between these procedures. Therefore, the decoding module must be selected according to the implementation mode of optical array logic.

\section{F. Possible Versions of the OPALS}

Various versions of the OPALS can be designed by properly connecting functional modules. Figure 11 shows three systems: Fig. 11(a) is a cascade system capable of executing real-time processing and control, 


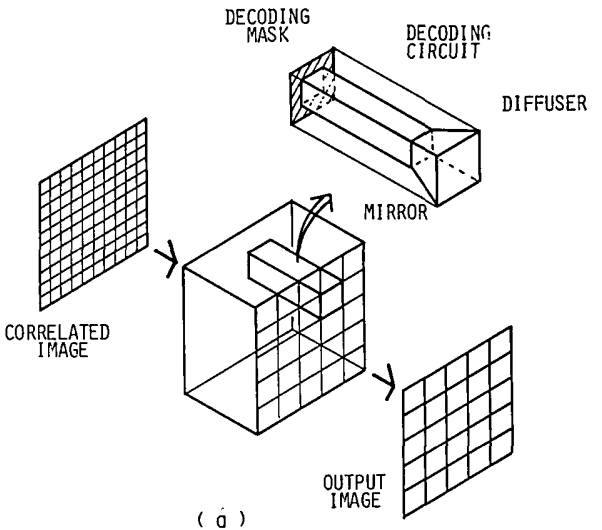

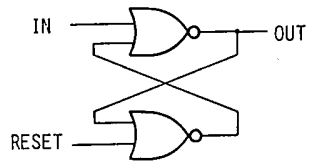

(b)

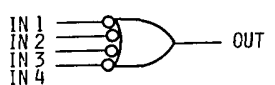

(c)
Fig. 10. Decoding module: (a) structure, (b) equivalent decoding circuit for optical array logic on a sequential processing mode, (c) that on a parallel processing mode.

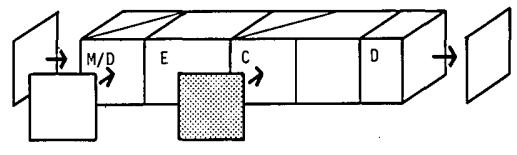

(a)

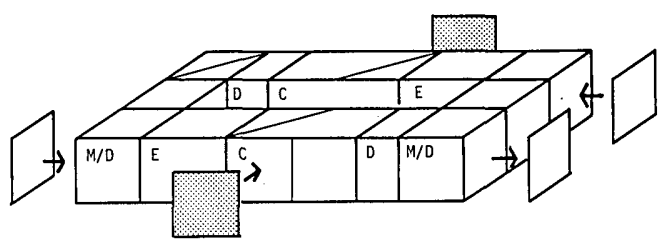

(b)

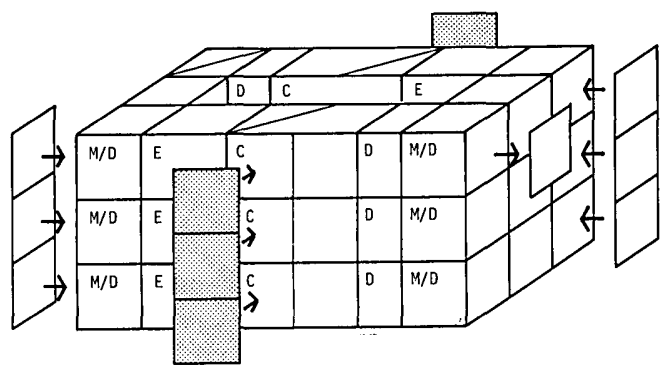

(c)

Fig. 11. Some examples of the modularized OPALS: (a) cascade system, (b) iterative system operated on a sequential processing mode, (c) iterative system on a parallel mode.

Fig. 11(b) is an iterative system operating in a sequential processing mode, and Fig. 11(c) is an interative system operating in a parallel processing mode. Note that, although systems operating in the sequential processing mode are inferior to those in the parallel mode in performance, they are superior in simplicity and

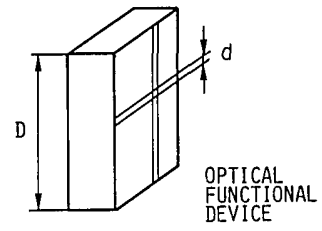

(a)

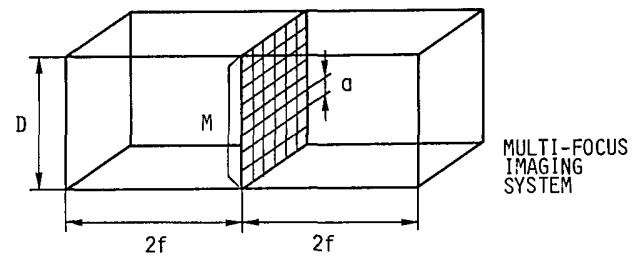

(b)

Fig. 12. Simplified model of (a) optical device and (b) optical system for estimating performance of the system.

cost. Simplified systems are often sufficient for special purposes.

The most salient feature of the modularized OPALS is that various architectures can be configured by combining the functional modules. When other functional modules, such as 2-D memory module, global interconnection module, input/output module, are considered, a more flexible computing system is possible.

\section{Performance of the Modularized OPALS}

Performance of the modularized OPALS is evaluated by the processing speed. First, we calculate the number of pixels in the OPALS, limited by the spatial resolution of an optical functional device and the optical system in a correlation module. Figure 12 depicts a simplified model of an optical device and an optical system. A square type of optical functional device and a multifocus imaging system are assumed.

The pixel number $N_{d v}^{2}$ on an optical functional device is

$$
N_{d v}^{2}=D^{2} / 4 d^{2},
$$

where $D$ and $d$ are the side length of an optical functional device and that of a pixel element, respectively; and the factor of 4 in the denominator is introduced by the coding method used in optical array logic.

On the other hand, the spatial resolution of a multifocus imaging system, $\mu$, is estimated by

$$
\mu=2 \lambda f / a=2 \lambda f M / D,
$$

where $\lambda$ is the wavelength of light, $f$ and $a$ are the focal length and side length of an imaging lens, respectively; and $M$ is the number of imaging lenses on a side of a lens array. Thus the pixel number $N_{o p}^{2}$ dealt with in the optical system is

$$
N_{o p}^{2}=D^{2} / 4 \alpha^{2} \mu^{2}=D^{4} / 16 \alpha^{2} \lambda^{2} f^{2} M^{2},
$$

where the factor of 4 in the denominator is again related to the coding method of the optical array logic, and 
Table I. Estimation of System Performance of the OPALS

\begin{tabular}{lcccc}
\hline $\begin{array}{c}\text { Assumed optical } \\
\text { functional device }\end{array}$ & $\begin{array}{c}\text { Processing } \\
\text { number } \\
N^{2}\end{array}$ & $\begin{array}{c}\text { Neighborhood } \\
\text { area size } \\
M^{2}\end{array}$ & $\begin{array}{c}\text { Processing } \\
\text { cycle } \\
\nu\left(\mathrm{s}^{-1}\right)\end{array}$ & $\begin{array}{c}\text { System } \\
\text { performance } \\
P(\text { AND/s) }\end{array}$ \\
\hline LCLV & $400^{2}$ & $26^{2}$ & 66 & $7.14 \times 10^{9}$ \\
Optical bistable device & $100^{2}$ & $26^{2}$ & $1 \times 10^{6}$ & $6.76 \times 10^{12}$ \\
Optoelectronic hybrid device & $500^{2}$ & $50^{2}$ & $1 \times 10^{6}$ & $6.25 \times 10^{14}$ \\
All-optical device & $1000^{2}$ & $100^{2}$ & $1 \times 10^{9}$ & $1.00 \times 10^{19}$ \\
\hline
\end{tabular}

$\alpha$ is a margin factor for avoiding crosstalk, normally set for 2 .

To achieve an efficient system, $N_{d v}$ should be equal to $N_{o p}$. When $N_{d v}=N_{o p}$, the following relation is obtained:

$$
M=d D / 2 \alpha \lambda f .
$$

This relation is useful for designing functional modules. $M$ is concerned with the size of a neighborhood region, which consists of $M \times M$ pixels centering on the $i j$ pixel. Optical array logic can handle pixels in the neighborhood region simultaneously as a parallel neighborhood operation of Eq. (1), so that as $M$ increases a greater number of pixels can be processed by the operation.

The performance of a set of the modularized OPALS (mixing/distributing, encoding, correlation, and decoding modules), $P$, is evaluated by

$$
P=\nu M^{2} N^{2} \text { [AND operation/s], }
$$

where $\nu$ and $N^{2}$ are the processing cycle of the system and pixel number, respectively. The unit of the performance is the number of AND operations per second, because the AND operation is a basic operation in the OPALS. Table I shows estimated values of performance of the OPALS when using different kinds of optical functional device. These values indicate the great ability of this modularized OPALS.

Operation of multiple sets of the modularized OPALS in parallel can increase the system performance. The system shown in Fig. 11(c) executes six times as many operations as estimated in Table $I$. Moreover, wavelength multiplexing ${ }^{13}$ can also improve the system performance.

\section{Conclusion}

We have considered several problems in constructing an optical computer and have proposed a new concept of an optical-digital computer based on modularization of the OPALS. Also, performance of the proposed system has been evaluated in terms of processing speed. The system is expected to have high performance when using multiple sets of the modularized OPALS together with wavelength multiplexing. Considering the capability of the modularized OPALS, we conclude that an optical computer based on the modularized OPALS is worthy of study as a next generation computing system.

\section{References}

1. A. A. Sawchuk and T. C. Strand, "Digital Optical Computing," Proc. IEEE 72, 758 (1984).

2. A. Huang, "Architecture Considerations Involved in the Design of an Optical Digital Computer," Proc. IEEE 72, 780 (1984).

3. A. W. Lohmann, "What Classical Optics Can Do for the Digital Optical Computer," Appl. Opt. 25, 1543 (1986).

4. T. E. Bell, "Optical Computing: A Field in Flux," IEEE Spectrum 23(8), 34 (1986).

5. A. Huang, "Design for an Optical General Purpose Digital Computer," Proc. Soc. Photo-Opt. Instrum. Eng. 232, 119 (1980).

6. A. Huang. "Parallel Algorithms for Optical Digital Computers," in Proceedings, Tenth International Optical Computing Conference (MIT Press, Cambridge, MA, 1983), pp. 13-17.

7. J. Tanida and Y. Ichioka, "OPALS: Optical Parallel Array Logic System,” Appl. Opt. 25, 1565 (1986).

8. G. R. Knight, "Interface Devices and Memory Materials," in Optical Information Processing Fundamentals, S. H. Lee, Ed. (Springer-Verlag, Berlin, 1981), pp. 111-179.

9. S. Hirose, T. Nishimura, K. Sugahara, S. Kusunoki, Y. Akasaka, and N. Tsubouchi, "A 10-Bit Linear Image Sensor Fabricated in Double Active Layers," in Technical Digest, 1985 Symposium on VLSI Technology, Kobe (May 1985), pp.34-35.

10. J. Tanida and Y. Ichioka, "Optical-Logic-Array Processor Using Shadowgrams. III. Parallel Neighborhood Operations and an Architecture of an Optical Digital-Computing System," J. Opt. Soc. Am. A 2, 1245 (1985).

11. K. Preston, Jr., and M. J. B. Duff, Modern Cellular Automata. Theory and Applications (Plenum, New York, 1984).

12. H. Fleisher and L. I. Maissel, "An Introduction to Array Logic," IBM J. Res. Dev. 19, 98 (1975).

13. J. Tanida and Y. Ichioka, "Optical Parallel Array Logic System. 2: A New System Architecture Without Memory Elements," Appl. Opt. 25, 3751 (1986). 